\title{
COMPORTAMENTO DO FOGO EM UM ANO CRÍTICO NAS UNIDADES DO INSTITUTO FLORESTAL DE SÃO PAULO
}

Jair Rosas da Silva ${ }^{1}$

\begin{abstract}
RESUMO
O estudo trata de avaliação da reação do fogo por meio de análise de regressão entre parâmetros representativos dos incêndios assinalados nas unidades do Instituto Florestal de São Paulo durante um ano crítico de incidência de incêndios, com o objetivo de propor modelos matemáticos capazes de exprimir o comportamento do fogo. $\mathrm{O}$ ano de 1988 foi considerado um dos anos mais críticos quanto à incidência de incêndios florestais durante os últimos cinqüenta anos. A extensão em área queimada demonstrou constituir-se em variável matemática dependente da duração do incêndio, refletindo melhor o comportamento do fogo nas unidades de produção que nas de conservação. Ocorreu associação entre duração do incêndio e umidade relativa do ar, segundo modelo polinomial logarítmico. Observou-se correspondências funcionais entre área queimada e tempo de duração do fogo com relação ao número de combatentes, atribuídas à severidade da estação de fogo, consoante modelos polinomiais de segundo grau.

Palavras-chave: incêndios florestais, modelos matemáticos, avanço do fogo, planejamento de controle
\end{abstract}

\section{THE FIRE BEHAVIOR IN A SEVERE WILDFIRE YEAR RECORDED IN SAO PAULO FORESTRY INSTITUTE STATIONS}

\begin{abstract}
This paper presents fire behavior based on some regression analysis among parameters of wildfire occurrences during a severe wildfire year in Sao Paulo Forestry Institute network, Brazil. The 1988 entire year was a severe fire season during the last fifty years in Sao Paulo State, when was burned 1,085.42 hectares. The extension of the burned area was a mathematical dependent variable of duration of fire, expressing the closest behaviour since it was related to the forestry production units rather to the conservation ones, due to available resources. An association also occurred between duration of fire and air humidity, following a logarithmic-polynomial model. A mathematical correlation took place as well between the burned area and the firefighting personnel, supposedly in conection with the severity of that related fire season. A correlation have also occurred between the duration of fire and the firefighting personnel, according to a polynomial model, considering all the forest units that were working towards wildfire control that year.

Keywords: forest fire, mathematical model, fire advance, fire control planning.
\end{abstract}

\section{INTRODUÇÃO}

O ano de 1988 foi particularmente crítico quanto à incidência de incêndios florestais nas unidades do Instituto Florestal do Estado de São Paulo. Relatórios indicam que condições anormais de clima, com duas fortes geadas no final de julho e seca bastante pronunciada de julho a outubro trouxeram como conseqüência o aparecimento de um conjunto de fatores que condicionaram a ocorrência de incêndios florestais.

Comportamento do fogo é o resultado da interação entre clima e condições do combustível, topografia, técnica de queima e forma de ignição. O seu conhecimento é útil para o planejamento da supressão, segundo Batista \& Soares (1997). Referidos autores estudaram o comportamento do fogo sob queimas controladas em povoamentos de Pinus taeda Linneaus no Estado do Paraná, baseandose em modelos existentes, segundo os quais a intensidade do fogo é o resultado do produto entre o calor de combustão, a massa de combustível disponível e a velocidade de propagação do fogo. Andrews (1986) reuniu vários métodos de cálculo de predição de incêndios florestais, incluindo tabelas, gráficos,

\footnotetext{
${ }^{1}$ Eng. Agr., Dr., Estação Experimental de Itapetininga, Instituto Florestal, CINP, Secretaria do Meio Ambiente do Estado de São Paulo. Caixa Postal 143, 18200-000 Itapetininga, SP. jairrosas@zipmail.com.br
} 
nomogramas, diagramas, multiplicadores e cálculos vetoriais, todos capazes de fornecer elementos que podem ser estimados, a saber: área, perímetro, probabilidades e componentes de ignição, taxa de propagação, energia calorífica liberada, intensidade da reação química, razão comprimento/largura do foco, altura de crestamento, distância de propagação da frente de avanço e da retaguarda, máxima distância de marcação de aceiros, controle do fogo sob ataque direto e indireto, teor de água do combustível considerado inerte e modelos de combustíveis habituais.
O presente trabalho teve o objetivo de estabelecer modelos matemáticos capazes de exprimir o comportamento do fogo em situações reais, contribuindo para o delineamento de diretrizes destinadas a nortear futuros programas de prevenção e combate aos incêndios florestais.

\section{MATERIAIS E MÉTODOS}

Os incêndios registrados em 1988 na rede de unidades do Instituto Florestal do Estado de São Paulo e as respectivas características de ocorrência são apresentados no quadro 1.

Quadro 1: Características dos incêndios ocorridos nas unidades do Instituto Florestal de São Paulo

Table 1: Features of the 1988's wildfires occurred in Sao Paulo Forestry Institute stations

\begin{tabular}{|c|c|c|c|c|c|c|c|}
\hline $\begin{array}{l}\text { Unidade administrativa } \\
\text { do Instituto Florestal }\end{array}$ & $\begin{array}{c}\text { Data de } \\
\text { ocorrência }\end{array}$ & $\begin{array}{c}\text { Área } \\
\text { atingida(ha) }\end{array}$ & $\begin{array}{l}\text { Duração do } \\
\text { fogo (min.) }\end{array}$ & $\begin{array}{c}\text { Início do fogo } \\
\text { (horário) }\end{array}$ & $\begin{array}{c}\text { Umidade } \\
\text { relativa ar }(\%)\end{array}$ & $\begin{array}{c}\text { Comba- } \\
\text { tentes }\end{array}$ & $\begin{array}{c}\text { Classe } \\
(*)\end{array}$ \\
\hline E. Exp. Itapetininga & Jan, 18 & 25,00 & 540 & 14 & - & 15 & III \\
\hline E. Ecol. Á. S. Bárb. & Jun, 07 & 24,20 & 240 & 20 & - & 6 & III \\
\hline E. Ecol. Itirapina & Jun, 13 & 6,00 & - & - & - & - & III \\
\hline E. Exp. Parag. Paul. & Jun, 28 & 2,00 & 540 & 12 & 60 & 8 & II \\
\hline E. Ecol. Itirapina & Jul, 06 & 4,00 & - & - & - & - & II \\
\hline E. Ex. B. Quirino & Jul, 16 & 60,00 & 420 & 15 & - & 30 & IV \\
\hline E. Exp. Pederneiras & Jul, 25 & 1,00 & 60 & 16 & 28 & 20 & II \\
\hline E. Exp. Itirapina & Jul, 27 & 5,00 & 240 & 14 & - & 20 & III \\
\hline E. Exp. Itirapina & Ago, 01 & 3,00 & 540 & 09 & - & 20 & II \\
\hline E. Exp. Assis & Ago, 05 & 7,00 & 105 & 15 & 38 & 40 & III \\
\hline E. Exp. Pederneiras & Ago, 07/08 & 31,00 & 1.950 & 14 & 26 & 35 & III \\
\hline E. Exp. Parag. Paul. & Ago, 07 & 5,00 & 60 & - & - & - & III \\
\hline E. Exp. Assis & Ago, 09 & 4,00 & 15 & 15 & 41 & 32 & II \\
\hline E. Exp. Assis & Ago, 09 & 0,20 & 35 & 14 & 41 & 2 & II \\
\hline E. Ex. S. J. R. Preto & Ago, 14 & 3,00 & 190 & 18 & - & 5 & II \\
\hline E. Ecol. Itirapina & Ago, 19 & 250,00 & - & - & - & - & $\mathrm{V}$ \\
\hline E. Exp. Pederneiras & Ago, 20 & 2,00 & 120 & 15 & 23 & 20 & II \\
\hline Fl. Prod. Batatais & Ago, 20 & 15,20 & 270 & 13 & 29 & 5 & III \\
\hline E. Exp. Araraquara & Ago, 21 & 0,10 & 30 & - & - & - & II \\
\hline E. Exp. Jaú & Ago, 22 & 16,90 & 240 & - & - & - & III \\
\hline E. Ecol. Itapeva & Ago, 22 & 0,02 & 45 & 20 & 70 & 9 & I \\
\hline E. Exp. Pederneiras & Ago, 25 & 2,00 & 240 & 18 & 15 & 20 & II \\
\hline E. Ex. S. J. R. Preto & Ago, 27 & 20,00 & 240 & 14 & - & 20 & III \\
\hline E. Ex. S. J. R. Preto & Ago, 27 & 5,00 & 150 & 15 & - & - & III \\
\hline Fl. Prod. Avaré & Ago, 27/28 & 2,00 & 720 & 17 & - & 17 & II \\
\hline E. Exp. Bauru & Ago, 27 & 1,00 & 90 & 15 & - & 10 & II \\
\hline E. Ex. S. J. R. Preto & Ago, 29 & 8,00 & 165 & 10 & - & 6 & III \\
\hline E. Exp. Itirapina & Ago, 31 & 49,00 & - & - & - & - & IV \\
\hline Pq Est. Cantareira & Set, $01 / 02$ & 0,30 & 300 & 21 & - & 30 & II \\
\hline E. Exp. Pederneiras & Set, 03 & 1,00 & 60 & 14 & 41 & 12 & II \\
\hline Pq E. S. Mar/Cunha & Set, $11 / 14$ & 54,00 & 3.840 & 20 & - & 43 & IV \\
\hline Pq Est. Cantareira & Set, $11 / 14$ & 375,00 & 4.560 & 10 & - & 320 & V \\
\hline Pq Est. T. A. Rib. & Set, $13 / 14$ & 15,00 & 1.680 & 09 & - & 32 & III \\
\hline E. Exp. Pederneiras & Set, 22 & 37,00 & 420 & 14 & 20 & 50 & III \\
\hline E. Exp. Pederneiras & Set, 24 & 2,00 & 120 & 14 & 15 & 15 & II \\
\hline E. Exp. Assis & Set, 27 & 0,30 & 15 & 06 & 70 & 3 & II \\
\hline E. Exp. Assis & Set, 27 & 0,20 & 15 & 06 & 70 & 2 & II \\
\hline E. Exp. Assis & Set, 27 & 0,50 & 20 & 05 & 66 & 8 & II \\
\hline E. Ecol. M. Guaçú & Set, 27 & 20,00 & - & - & - & - & III \\
\hline E. Ecol. M. Guaçú & Out, 01 & 20,00 & 150 & 20 & 38 & 35 & III \\
\hline E. Exp. Pederneiras & Out, 08 & 0,50 & 90 & 17 & 29 & 5 & II \\
\hline E. Ecol. M. Guaçú & Dez, 20 & 8,00 & 150 & 17 & 92 & 30 & III \\
\hline Total & 42 & 1085,42 & - & - & - & 925 & - \\
\hline
\end{tabular}

(*) Segundo Canadian Forestry Service (Ramsey \& Higgins apud Soares,1984). 
A análise de variância e avaliação de interdependência entre os valores obtidos das variáveis em estudo foi efetuada com emprego de regressão linear, conforme Ferreira (1996), utilizando-se o pacote estatístico SAS, com aplicação do teste de ' $t$ ' de Student, a 5\% e 1\% de significância e pesquisa de modelos de regressão para dados sem repetição. Os pontos de mínima e de máxima das funções polinomiais obtidas foram determinados por derivação, conforme Magalhães (1944).

\section{RESULTADOS E DISCUSSÃO}

\section{Interdependência entre área queimada e duração do incêndio:}

A análise de variância com emprego de regressão linear entre área total queimada e tempo de duração do incêndio foi significativa no nível de $99 \%$ de probabilidade. Desse modo, ocorreu associação entre os parâmetros pesquisados (figura 1).

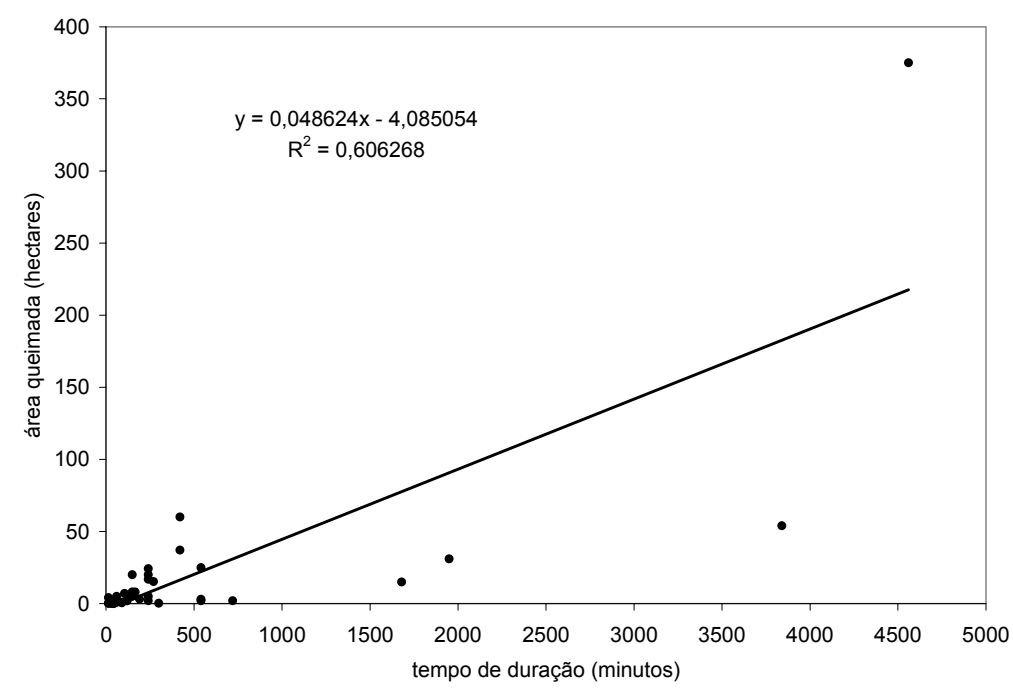

Figura 1: Representação da associação entre área total queimada (y) e duração do incêndio (x)

Figure 1: Graphic of the mathematical correlation between burned area (y) and duration of fire $(x)$

Tal resultado confirma a tendência ao inter-relacionamento entre os parâmetros avaliados, visto que o comportamento dos incêndios quanto a esses parâmetros nos eventos ocorridos em 1986 e 1987 nos parques estaduais da Instituição foi também identificado e condicionado por modelo linear não-logarítmico, conforme Silva (2000).

Desvios do modelo de regressão entre área queimada e duração do incêndio:

A correlação procura determinar quão bem uma equação linear, ou de outra espécie, descreve ou explica a relação entre variáveis, segundo Ferreira (1996). Considerando-se que a interdependência entre área queimada e duração do fogo é descrita pelo modelo anterior, segundo o coeficiente de correlação $\mathrm{R}=0,7786$, esse resultado indica que os desvios de regressão representam $22,14 \%$ das variáveis levantadas no estudo.
O comportamento matemático com menores afastamentos do modelo de regressão obtido ocorreu com o conjunto das unidades de produção, portanto, funcionando com melhor acerto e de forma mais previsível do que para o conjunto das unidades de conservação. Tal resultado pode ser atribuído aos recursos disponíveis nas unidades de produção, em termos de maquinário e equipamento, à experiência e conscientização do pessoal em atividade, às facilidades de acesso e às parcerias com empresas e órgãos da região.

\section{Associação entre duração do incêndio e umidade relativa do ar:}

Ocorreu correspondência funcional entre tempo de residência do fogo e umidade relativa do ar, no nível de $95 \%$ de probabilidade, segundo um coeficiente de determinação R2=0,2834 (figura 2). 


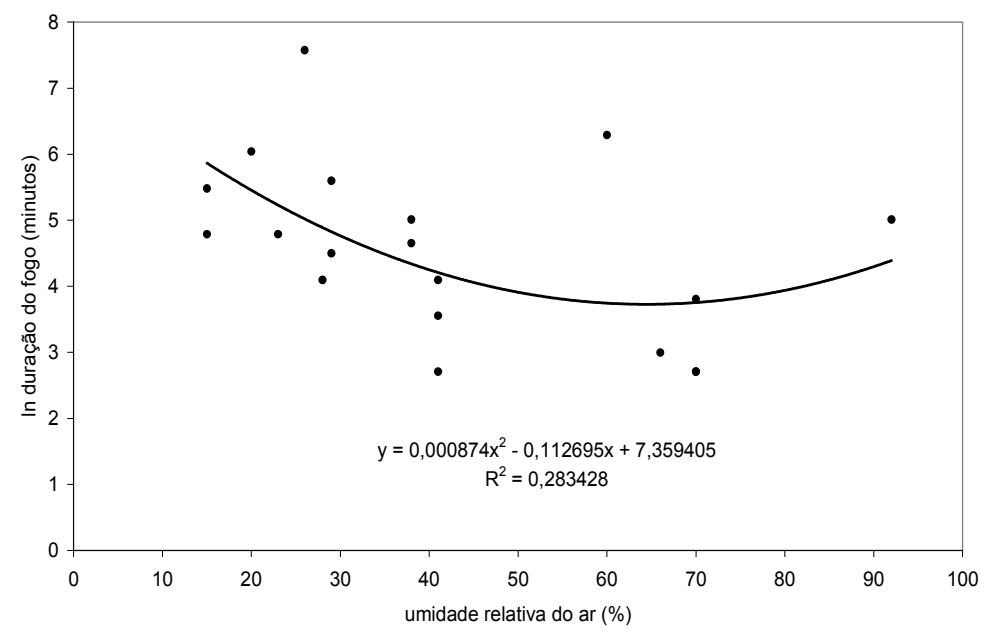

Figura 2: Associação entre ln tempo de duração do incêndio, em minutos e umidade relativa do ar.

Figure 2: Graphic of the mathematical correlation between duration of fire, in minutes and air humidity.

A função matemática obtida para representar o comportamento da variável dependente "tempo de duração do incêndio", em função da umidade relativa do ar, tem um ponto de mínima, correspondente ao valor de $\mathrm{x}=64,47 \%$, ponto em que espera-se que o tempo de residência do fogo seja mínimo, para as condições do estudo. A essa condição teórica ideal teria correspondido um período de residência do fogo de 41,54 minutos (41 minutos e 32 segundos).

\section{Associação entre área queimada e umidade relativa do ar:}

Não foi significativa a análise de variância com emprego de regressão efetuada entre totais em áreas queimadas e os respectivos valores de umidade relativa do ar, portanto, não ocorreu correspondência funcional entre esses parâmetros de comportamento do fogo.

Do ponto de vista prático esse resultado indica que em um ano crítico de incidência de incêndios, embora o tempo de residência do fogo tenha se comportado como função dependente da umidade relativa do ar, esse condicionante meteorológico não influenciou as extensões em áreas atingidas pelo fogo.

\section{Interdependência entre área queimada e número de combatentes:}

Ocorreu associação funcional entre área total queimada e número de pessoas que participou do combate direto aos incêndios, no nível de $99 \%$ de probabilidade nas condições do experimento (figura 3 ).

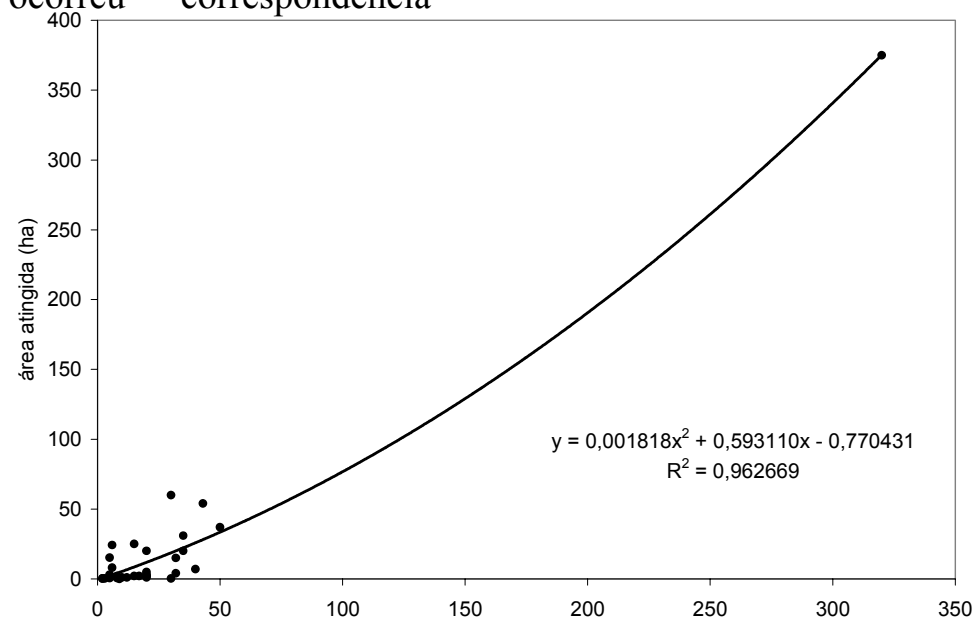

Figura 3: Associação entre área queimada, em hectaresmbatentênero de combatentes dos focos Figure 3: Graphic of the mathematical correlation between burned area and firefighting personnel 
O valor do coeficiente de determinação obtido nessa análise $(\mathrm{R} 2=0,9627)$ indica uma correspondência estreita. Considerando que esse parâmetro estatístico do modelo de regressão obtido situou-se nas proximidades da correlação absoluta, esse resultado indica a importância que representa o envolvimento de combatentes dos incêndios, evidenciando a influência do treinamento das brigadas e da organização estratégica das ações de combate.

$\mathrm{O}$ modelo obtido para o comportamento da variável dependente "área queimada" em função do número de combatentes tem um ponto de mínima, correspondente ao valor de $\mathrm{x}=163$ combatentes. Em um evento ocorrido em um ano crítico de incidência de fogo nas condições do estudo, em que a variável independente "número de combatentes" atingir esse valor, espera-se que a área total queimada seja mínima, correspondendo a uma área de 144,21 hectares.

\section{Interdependência entre duração do fogo e número de combatentes:}

Ocorreu associação entre os parâmetros tempo de duração do fogo e o respectivo número de combatentes dos focos de incêndio ocorridos nas unidades de produção e conservação do Instituto Florestal de São Paulo, durante o ano de 1988. Essa correspondência funcional, segundo um coeficiente de determinação R2 $=0,5980$, é mostrada graficamente na Figura 4.

A derivação do modelo matemático obtido indica que este tem um ponto de máxima correspondente a 322 combatentes em um evento de grandes proporções passível de ocorrer em um ano crítico de incidência de incêndios florestais, para as condições do estudo. De acordo com o modelo, esse contingente corresponderia a um período de residência do fogo de 4.566,295 minutos (76 horas e 06 minutos). Assim, o modelo reflete com propriedade as condições observadas no incêndio ocorrido em meados de setembro no Parque Estadual da Cantareira (Quadro 1).

\section{Interdependência entre número de combatentes e umidade relativa do ar:}

Não foi significativa a análise de regressão efetuada entre número de combatentes e umidade relativa do ar, no nível de $95 \%$ de probabilidade. Esse resultado indica a ausência de correlação entre esses dois parâmetros de comportamento do fogo em um ano considerado crítico, para as condições das unidades do Instituto Florestal de São Paulo.

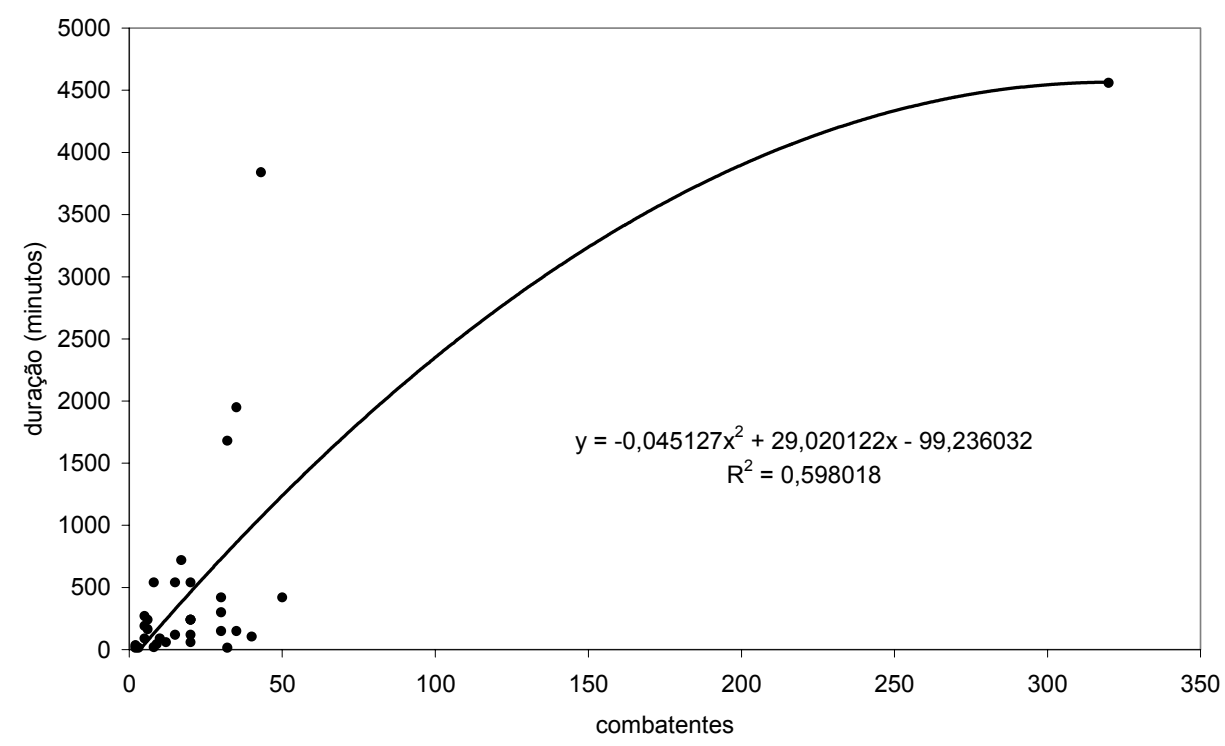

Figura 4: Associação entre duração do fogo, em minutos e número de combatentes dos focos

Figure 4: Graphic of the mathematical correlation between duration of fire and firefighting personnel 


\section{CONCLUSÃO}

Foi reafirmado ser a extensão de áreas atingidas por fogo uma variável dependente do tempo de duração dos incêndios.

O modelo matemático obtido para o comportamento do fogo em termos de áreas atingidas em função do tempo de duração dos incêndios funcionou mais estreitamente para o conjunto de unidades de produção do que para o conjunto das unidades de conservação, em função de disponibilidade de pessoal e equipamento.

Para as condições de um ano crítico em termos de incêndios nas unidades do Instituto Florestal de São Paulo, a extensão em áreas queimadas e o tempo de duração dos incêndios demonstraram constituírem-se em variáveis dependentes do número de combatentes, evidenciando a importância do treinamento e da organização das ações estratégicas de combate aos incêndios.

Os modelos matemáticos obtidos podem ser utilizados como ferramenta auxiliar no planejamento de controle e combate aos incêndios florestais nas unidades da Instituição pesquisada.

\section{REFERÊNCIAS}

ANDREWS, P. L. Methods for predicting fire behavior - you do have a choice. Fire Management Notes, v. 47, n. 2, p.6-10, 1986.

BATISTA, A.C.; SOARES, R.V. Avaliação do comportamento do fogo em queimas controladas sob povoamentos de Pinus taeda no norte do Paraná. Floresta, UFPR, v. 25, n. 1/2, p.31-42, 1997.

FERREIRA, P.V. Regressão e correlação. In: FERREIRA, P.V. Estatística experimental aplicada à agronomia. Maceió: Universidade Federal de Alagoas, 1996, p.489-530.

MAGALHÃES, P.A. O cálculo infinitesimal e algumas das suas inumeráveis aplicações: cálculo diferencial. Tomo 1. Rio de Janeiro: Escola Nacional de Agronomia, 1944. 330 p. (Série Didática, 6).

SILVA, J.R. Incêndios de 1986 e 1987 nos parques estaduais do Instituto Florestal de São Paulo. Revista do Instituto Florestal, v. 12, n. 1, p.1-12, 2000.

SOARES, R.V. Perfil dos incêndios florestais no Brasil em 1983. Brasil Florestal, v. 13, n. 58, p.31-42, 1984. 